\title{
Photo Privacy Protection on Online Social Networks
}

Yifang Li

Clemson University

Clemson, SC 29634, USA

yifang2@g.clemson.edu
Permission to make digital or hard copies of part or all of this work for personal or classroom use is granted without fee provided that copies are not made or distributed for profit or commercial advantage and that copies bear this notice and the full citation on the first page. Copyrights for third-party components of this work must be honored. For all other uses, contact the owner/author(s).

Copyright held by the owner/author(s).

CSCW'18 Companion, November 3-7, 2018, Jersey City, NJ, USA ACM 978-1-4503-6018-0/18/11.

https://doi.org/10.1145/3272973.3272988

\begin{abstract}
Through my doctoral research, I aim to provide a better online photo privacy protection strategy than existing approaches such as photo self-censorship and recipient control on Online Social Networks (OSNs). To inform the building for an effective and usable photo privacy protection system on OSNs, I gain understanding on the two parameters that influence photo privacy-photo content and recipientthrough a series of studies and provide design guidelines. My research will benefit privacy researchers, online social network designers, policymakers, computer vision researchers, and researchers in the field of CSCW who study collaborative photo privacy protection.
\end{abstract}

\section{Author Keywords}

Privacy; security; photo privacy; photo sharing; Online Social Network; social media; photo obfuscation

\section{Introduction and Research Questions}

Sharing photos on OSNs helps users manage the impression others have of them, maintain an off-line relationship with their family and friends, and gain attention from a wider audience than their existing friend circle [10]. While users enjoy these benefits, the rich visual information that photos contain may lead to privacy leakage, such as identification, location leakage, sensitive information leakage, and social activity leakage [1]. Privacy leakage could harm users' im- 


\section{Expected gain from attend ing the CSCW Doctoral Colloquium}

My proposal defense is scheduled for Fall 2018. I anticipate that by that time I will finish the on-going study which identifies photo sensitive content and users' sharing preference with different recipients, and propose my next study about users' other photo privacy protection schemes. I hope to take away constructive guidance and comments from senior researchers and fellow attendees for my next two studies from the perspectives of general directions to the methodology details, as well as helping me identify any gaps in my research thread. Eventually, my research results will be applied to Online Social Networks, hence the advice from CSCW researchers will be very valuable. pression management; furthermore, it may threaten their physical and property security [1]. Like in other similar situations (e.g., users self-censor posts and comments before posting them on Facebook [3]), to avoid these problems, users often turn to a self-censorship strategy [5]. Self-censorship as a strategy has a number of drawbacks. Users present a positive persona due to social pressure but fail to present a true self. Self-censorship also hampers the communicative ability of OSNs, so that OSNs risk becoming an asocial environment. Privacy mechanisms implemented by OSNs include controlling the photo recipients (e.g., Facebook audience selection), and controlling photo content disclosure which has been investigated in CSCW research (e.g., photo obfuscations [8]).

To ground our questions within a theoretical framework, we looked to both Contextual Integrity (CI) [9], a philosophical model of privacy, and a behavioral privacy model [2] that examines Privacy-Enhancing Behaviors (PEBs) and situates these within a temporal context. $\mathrm{Cl}$ identifies five relevant privacy parameters: sender, attribute (what type of information), subject (a particular information subject), recipient, and transmission principles [9]. In a photo privacy setting, this model may not be suitable because the attribute can only be visual content and the subject is primarily just the user him/herself. Moreover, photo content (e.g., a person's identity, activity, object, environment) is an important parameter that is not covered in $\mathrm{Cl}$ model but is accounted for in the behavioral model. Hence, we situate our work within the behavioral model. The behavioral model emphasizes two parameters-information recipient and information content [2]. It states that privacy can be achieved by adjusting either recipient or content [2]. All three photo privacy preserving schemes we identified in prior work (e.g., [3, 8]) -self-censorship, recipient control, content control- fit within the "avoidance" temporal category of PEB.
My research aims to optimize the behavioral privacy model in the context of photo privacy, study the two parameters content and recipient, offer an alternative photo privacy protection strategy other than self-censorship and OSN recipient control, and provide design guidelines for effective and usable online photo privacy protection systems. To achieve this goal, I plan to answer the following questions.

Study and select promising obfuscation methods (can be viewed as a part of the content parameter):

- What are the effective and usable obfuscations?

- As an extreme privacy protection scheme, is photo self-censorship prevalent? Can obfuscations combat it and encourage photo sharing?

Understand what to obscure (content) and prevent from whom (recipient):

- What is the sensitive content in photos to be obscured?

- With different groups of recipients, what are users' preferences of sharing different categories of sensitive content?

To create a more collaborative and usable privacy protection mechanism, I need to have a better understanding of users' current photo privacy protection behaviors:

- Other than identified self-censorship and recipient control that OSNs provide, are there other privacy protection schemes that they often use?

\section{Research Plan}

Identifying effective and usable obfuscations Focusing on controlling photo content disclosure, my first study introduces privacy-enhancing obfuscations and con- 


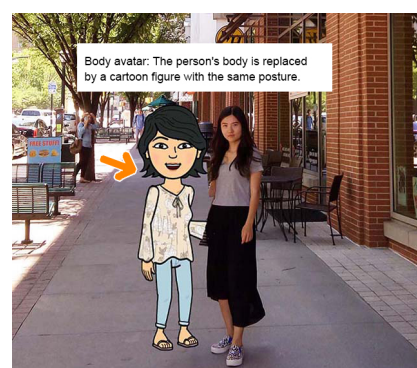

Figure 1: Avatar, replaces a person with an avatar, preserves the facial expression and body gesture, and hides the identity.

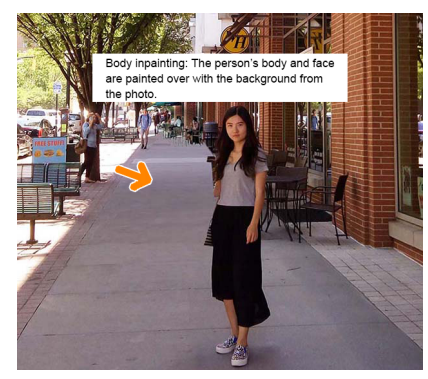

Figure 2: Inpainting, completely removes a person from a photo. duct an online experiment with 271 participants to evaluate their effectiveness against human recognition and how they affect the viewing experience. Results suggest that avatar (Figure 1) and inpainting (Figure 2) are two promising obfuscations which are effective and provide a good viewer experience, while the widely adopted blurring and pixelating are not effective. Two pilot studies are published in HFES [6] and CV-COPS [7]. The complete study is published and will be presented at CSCW 2018 [8].

Avatar and inpainting are robust when de-identifying both unfamiliar and familiar people

In this study, one limitation is that we only explored obfuscations' effectiveness for de-identifying unfamiliar people (people in stimuli were unknown to the participants). We conducted an experiment where participants identified both familiar and unfamiliar people applied different obfuscation methods. We find that avatar and inpainting are robust to the increased likelihood of recognition associated with familiarity so they would be useful privacy enhancement tools on OSNs. We are revising this manuscript.

\section{Obscuring scene elements}

My prior work only considers human as the most sensitive content to be obscured, however, various incidental information could also harm privacy, such as monitor or indoor scene. We studied 11 filters applied to obfuscate 20 different objects and evaluated how effectively they protect privacy and preserve image quality for human viewers. This work has been published and was presented at $\mathrm{CHI}$ 2018 [4].

Obfuscation may combat photo self-censorship We quantified the prevalence of self-reported photo selfcensorship and associated this with gender, age, and privacy preference. We find that over half of the participants have self-censored photos due to privacy concerns and privacy-conscious people were more likely to censor photos. Among the participants who reported they had selfcensored photos, half of them were willing to share the previously censored photo if they would be able to obfuscate portions of the photo to enhance privacy. Hence, privacypreserving obfuscations may be useful for combating photo self-censorship. The manuscript is in preparation.

Identifying sensitive content and users' sharing preference Focusing on the content parameter, we must know what portions are considered sensitive and should be obscured. We aim to 1) identify sensitive content in photos from a human-centered perspective and 2) study people's sharing preference of different sensitive content with 20 recipient groups (e.g., friend, colleague). We plan to provide a taxonomy of sensitive content categories associated with users' sharing preference with different recipients. We collected sensitive photos and/or descriptions of sensitive photos from 116 MTurk participants and asked them to identify which elements of the photo made each photo sensitive. Afterwards, they rated the likelihood to share this sensitive content with each of the 20 recipient groups. We have published the result from our pilot study in CV-COPS [5], and the main study is currently in progress.

Users' Photo Privacy Protection Schemes

This study will aim to understand users' privacy protection schemes other than self-censorship and recipient control. Through interviews with OSN users, we can understand the reasons they choose these schemes (e.g., convenient, reliable), identify the pros and cons, study if our obfuscation system can be a better alternative, and incorporate them into design recommendations to create a more collaborative and user-friendly system that encourages sharing. This work is scheduled for completion in February 2019. 


\section{Acknowledgements}

This research was supported by the National Science

Foundation under grant no.1527421. Any opinions, findings, and conclusions or recommendations expressed in this material are those of the authors and do not necessarily reflect the views of the National Science Foundation. I thank my advisor Dr. Kelly Caine for all her great support and suggestions on this project. I also thank Dr.

Bart Knijnenburg and our colleagues from the HATlab for suggestions that improved the studies. Additionally, I am grateful to the people who participated in the studies.
Photo Privacy Protection System Evaluation

After gaining sufficient knowledge from these studies, I plan to implement the photo privacy protection system on Facebook, recruit OSN users to upload their own photos and use our system, then test its effectiveness and usability.

This work is scheduled for completion in August 2019.

\section{Expected Contributions}

By answering all research questions above, we can have a deeper understanding of the two parameters-photo content and recipient, propose a better alternative for photo privacy protection on OSNs which preserves the privacy and encourages sharing, and provide design guidelines for developing effective and collaborative systems. My work may be useful to privacy researchers, online social network designers, policy makers, and researchers in the field of CSCW who study the collaborative photo privacy protection.

\section{REFERENCES}

1. Shane Ahern, Dean Eckles, Nathaniel S Good, Simon King, Mor Naaman, and Rahul Nair. 2007.

Over-exposed?: privacy patterns and considerations in online and mobile photo sharing. In Proceedings of the SIGCHI conference on Human factors in computing systems. ACM, 357-366.

2. Kelly Caine. 2009. Exploring everyday privacy behaviors and misclosures. Georgia Institute of Technology.

3. Sauvik Das and Adam Kramer. 2013. Self-Censorship on Facebook. In Proceedings of the International Conference on Weblogs and Social Media (ICWSM).

4. Rakibul Hasan, Eman Hassan, Yifang Li, Kelly Caine, David J Crandall, Roberto Hoyle, and Apu Kapadia. 2018. Viewer experience of obscuring scene elements in photos to enhance privacy. In Proceedings of the
2018 CHI Conference on Human Factors in Computing Systems. ACM, 47.

5. Yifang Li, Wyatt Troutman, Bart P. Knijnenburg, and Kelly Caine. 2018. Human perceptions of sensitive content in photos. In Computer Vision and Pattern Recognition Workshops (CVPRW). IEEE.

6. Yifang Li, Nishant Vishwamitra, Hongxin Hu, Bart P Knijnenburg, and Kelly Caine. 2017a. Effectiveness and users' experience of face blurring as a privacy protection for sharing photos via online social networks. In Proceedings of the Human Factors and Ergonomics Society Annual Meeting, Vol. 61. SAGE, 803-807.

7. Yifang Li, Nishant Vishwamitra, Bart P. Knijnenburg, Hongxin Hu, and Kelly Caine. 2017b. Blur vs. Block: Investigating the Effectiveness of Privacy-Enhancing Obfuscation for Images. In Computer Vision and Pattern Recognition Workshops (CVPRW). IEEE.

8. Yifang Li, Nishant Vishwamitra, Bart P. Knijnenburg, Hongxin $\mathrm{Hu}$, and Kelly Caine. 2017c. Effectiveness and users' experience of obfuscation as a privacy-enhancing technology for sharing photos. Proceedings of the ACM on Human-Computer Interaction 1, 2 (2017).

9. Helen Nissenbaum. 2009. Privacy in context: Technology, policy, and the integrity of social life. Stanford University Press.

10. Anne Oeldorf-Hirsch and S Shyam Sundar. 2010. Online photo sharing as mediated communication. In annual conference of the International Communication Association, Singapore. 